\title{
Extragalactic Globular Clusters in the Near-Infrared
}

\author{
Maren Hempel ${ }^{1}$, Markus Kissler-Patig ${ }^{1}$, Michael Hilker ${ }^{2}$, Thomas H. Puzia ${ }^{3}$, \\ Jean P.Brodie ${ }^{4}$, Paul Goudfrooij ${ }^{5}$, Dante Minniti ${ }^{6}$, and Stephen E.Zepf ${ }^{7}$ \\ 1 European Southern Observatory, Garching 85748, Germany \\ 2 Sternwarte Bonn, Germany \\ 3 Sternwarte der Ludwig-Maximilians Universität, München, Germany \\ 4 UCO/Lick observatory, University of California, Santa Cruz, USA \\ ${ }^{5}$ Space Telescope Science Institute, Baltimore, US \\ 6 P. Universidad Católica, Santiago, Chile \\ 7 Yale University, New Haven, USA
}

\begin{abstract}
Visual and near-infrared colours are used to identify intermediate-age globular cluster in early-type galaxies. In NGC 5846 and NGC 4365 colour-colour diagrams (V-I vs. V-K) reveal a sub-population in the globular cluster systems with an age much younger than the expected old populations (13-15 Gyr). A comparison with SSP models dates the formation of this younger population 2-7 Gyr back. Further evidence for multiple star formation events is given by the age distribution in both systems and the comparison to models. Our photometric age determination was confirmed by spectroscopy for a small sample of the clusters in NGC 4365.
\end{abstract}

\section{Introduction}

Globular cluster are known to form during major star formation episodes [1], [2]. Consequently the star formation history in galaxies can coarsely be described by the age structure of the globular cluster system. The detection of sub-populations and the determination of their ages is therefore of great interest in galaxy formation studies. But besides the pure detection of intermediate-age populations the importance of recent star formation events for the galaxy evolution has to be considered. Using combined optical and near-infrared photometry provides the observational tools for this task. Taking advantage of the different sensitivity to age and metallicity of colours in the visual and near-infrared bands [3] the degeneracy of both parameters [4] can be lifted. Photometry as opposite to spectroscopy allows us to quantify the age distribution by including a large number of objects within the globular cluster system. The draw back is photometry being a somewhat less accurate age indicator and less informative on the chemistry. First results of this approach towards age determination and identification of multiple star formation events have been published by Kissler-Patig et al. [5] and Puzia et al. 33. It is highly encouraging that the photometric results have recently been confirmed by spectroscopy [6]. The results presented here are part of this ongoing project on globular cluster systems of early-type galaxies. 


\section{The Project}

Our complete galaxy sample consists of 11 early-type galaxies covering a wide range of luminosities in various environments, e.g. central cluster or group galaxies (NGC 1399, M87, NGC 5846) and isolated ellipticals (NGC 7192, NGC 3115, NGC 1426). In this study we combine near-infrared data $\left(\mathrm{K}_{s}\right.$-band, obtained with ISAAC) and optical (B-, V-and I-band, WFPC2/HST archive) photometry. The observed ISAAC fields were chosen to match the HST archive data where the PC chip was centred on the galaxy. The data have been reduced in the standard way using IRAF and SExtractor [7] as described in [5] and [3].

\section{$3 \quad$ Results}

\subsection{Colour Distributions}

Intermediate age populations can be identified by using in colour-colour diagrams, e.g. (V-I) vs. (V-K). Examples of these are given in Fig. 1. Hereby it is important that the different completeness levels for optical and near-infrared colours are taken into account. A limit in photometric errors (0.15 mag in V-I) is used as additional selection criterion. In order to exclude background galaxies we used the photometric FWHM estimated by SExtractor and compared it with the one we obtained for point sources.

In the galaxies shown here (NGC 4365 and NGC 5846) we find a distinct second red population (indicated by small ellipses) of globular clusters which are red in $(\mathrm{V}-\mathrm{K})$ with $(\mathrm{V}-\mathrm{K}) \in\langle 2.9,3.2\rangle$ but intermediate in $(\mathrm{V}-\mathrm{I})$ with $(\mathrm{V}-\mathrm{I}) \in\langle 1.0,1.2\rangle$.
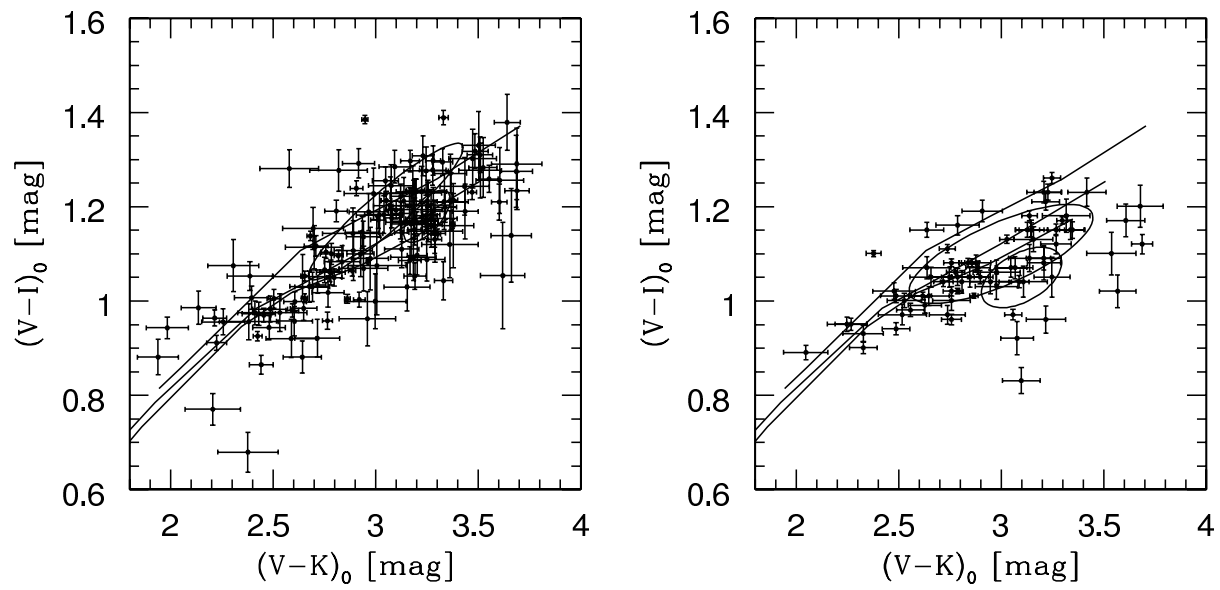

Fig. 1. (V-I) vs. (V-K) colour-colour diagram for NGC 5846 and NGC 4365. The solid lines mark the isochrones for a 15, 5 and 3 Gyr SSP model. The ellipses are eye guides to the two identified red populations. 


\subsection{Age Distribution}

Our age determination of globular clusters relies on the comparison of SSP isochrones with colour-colour diagrams of globular cluster systems. Various single stellar population models (SSP) built by Bruzual \& Charlot 8], Maraston [9], Vazdekis [10] or Kurth et al. [11] are available. For the work discussed here the model isochrones by Bruzual \& Charlot are used. Given the uncertainty in the isochrone modelling $( \pm 3 \mathrm{Gyr})$ we do not intend to determine absolute ages. The goal is rather to identify possible sub-populations and to obtain their relative ages. A first attempt to distinguish the age structure of globular cluster systems in different environment is given in Fig. 2. This figure shows our quantitative method to detect intermediate age populations in globular cluster systems, supplementing the purely visual inspection of colour-colour diagrams. Ages for each cluster are determined in the colour-colour diagrams with respect to the model isochrones. The $50 \%$ level is marked by a solid line. For comparison we modelled the colour-colour diagrams for a pure $15 \mathrm{Gyr}$ old population and a mixed system respectively, consisting of 50\% $15 \mathrm{Gyr}$ and 50\% 1 Gyr old globular clusters. The results were than evaluated in the same way as the observed data.
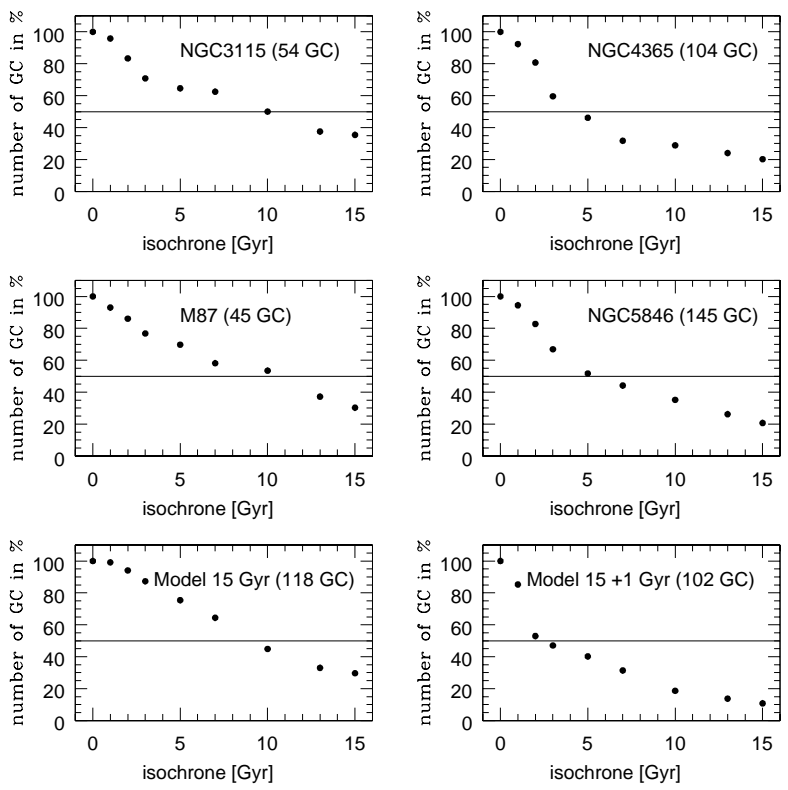

Fig. 2. Age distribution for GCS in different galaxies (NGC 5846, NGC 4365, M87 and NGC 3115). Each panel shows the cumulative age distribution in a given galaxy, the total number of objects is given in brackets. The two lower panels show the distributions for artificial data (left: a purely 15 Gyr old and right: 50\% 15 Gyr and 50\% 1 Gyr). 
As shown in Fig.2, M87 and NGC 3115 have age distributions compatible to a pure old systems [3], 12 (see lower left panel) with $\sim 50 \%$ of clusters lying above the 10 Gyr isochrone. NGC 5846 and NGC 4365 show a steep drop in numbers between 1 and $5 \mathrm{Gyr}$ and reach the $50 \%$ level already at an age of about 5 Gyr or younger. This is consistent with the presence of a significant fraction of younger objects.

\section{Conclusion}

The results presented here show that combined optical and near-infrared photometry is a very promising method to detect intermediate-age stellar populations. Given that already 2 out of 6 galaxies show a significant intermediate-age population this might revive the discussion on when ellipticals formed the bulk of their stars.

\section{References}

1. Holtzman J.A., Faber S.M. et al.: AJ 103, 691 (1992)

2. Kissler-Patig M.: RvMA 13, 13 (2000)

3. Puzia Th.H., Zepf S.E., Kissler-Patig M. et al.: A\&A 391, 453 (2002)

4. Worthey G.: ApJS 95, 107 (1994)

5. Kissler-Patig M., Brodie J.P., Minniti D.: A\&A 391, 441 (2002)

6. Larsen S.: ApJ submitted

7. Bertin E. \& Arnouts S.:A\&AS 117, 393 (1996)

8. Bruzual A.G., Charlot S.: (2000) based on 1996, electronically available see: Leitherer C. et al.: PASP 108, 996 (1996)

9. Maraston C.: priv. communication, based on Maraston C. MNRAS 370, 872 (1998)

10. Vazdekis A.: ApJ 513, 224 (1999)

11. Kurth O.M., Fritze-v.Alvensleben U., \& Fricke K.J.: A\&AS 138, 59 (1999)

12. Jordán A., Cóte P., West M.J et al.: ApJ 576, L113 (2002) 